\title{
¿Responden las investigaciones en las áreas protegidas de Risaralda a las necesidades de manejo y gestión de la biodiversidad?*
}

\section{Does Research on the Protected Areas in Risaralda Answer to the Biodiversity Management Needs?}

\section{Les recherches sur les zones protégées de la Région du Risaralda (Colombie) répondent-t-elles aux besoins de gérance et gestion de la biodiversité?}

\author{
Lina Cristina Vásquez-Uribe**, Clara Lucía Matallana-Tobón**
}

Recibido: 2015-03-18 // Aprobado: 2015-06-20 // Disponible en linea: 2016-01-30

Como citar este artículo: Vásquez Uribe L. C. y Matallana Tobón, C. L. (2016). ¿Responden las investigaciones en las áreas protegidas de Risaralda a las necesidades de manejo y gestión de la biodiversidad? Ambiente y Desarrollo, 20(38), 27-40. http://dx.doi.org/10.11144/Javeriana. ayd20-38.riap

doi:10.11144/Javeriana.ayd20-38.riap

\begin{abstract}
Resumen
El objetivo de este estudio fue evaluar la incorporación de los resultados de las investigaciones en el manejo y gestión de las áreas protegidas de Risaralda. Para ello, se recopiló información sobre investigaciones realizadas en tres parques nacionales naturales y tres parques regionales naturales. Como resultados, la mayoría fueron estudios en Otún-Quimbaya, están en revistas indexadas y tratan sobre especies vegetales y animales. Todos los planes de manejo cuentan con un programa de investigación científica, y se propusieron nueve indicadores, relacionados con: existencia, actualización, investigación, pertinencia, incorporación del plan de manejo y el tipo de investigación. Se concluye que es fundamental fortalecer centros de documentación, establecer alianzas con universidades e institutos de investigación, definir líneas de investigación de cada área y aplicar un sistema de indicadores.
\end{abstract}

Palabras clave: indicadores; investigación aplicada; conservación; prioridades

\footnotetext{
Este artículo es resultado de investigación de la línea Áreas Protegidas y Estructuración Ecológica del Territorio, del Instituto de Investigación de Recursos Biológicos Alexander von Humboldt.

** Bióloga de la Pontificia Universidad Javeriana, Bogotá, Colombia. Correo electrónico: linav15@gmail.com

*** Ecológa, MSc. Biología de la Conservación de la Universidad Estatal de California, investigadora adjunta del Instituto de Investigación de Recursos Biológicos Alexander von Humboldt, Bogotá, Colombia. Correo electrónico: cmatallana@humboldt.org.co
} 


\begin{abstract}
The purpose of this study was to assess the inclusion of the results from research works in the management of protected areas in Risaralda. For this purpose we collected information on research projects carried out in three national natural parks and in three regional natural parks. As a result we could see most of the studies were carried out in Otún-Quimbaya, were published in indexed journals and dealt with plant and animal species. All management plans have a scientific research project and we proposed nine indicators related to the following: existence; updates; research; appropriateness; inclusion of the management plan; and the type of research. We concluded that it is of vital importance strengthening the documentation centers, establishing alliances with universities and research institutes, defining research lines for each area, and applying a system of indicators.
\end{abstract}

Keywords: indicators; applied research; preservation; priorities

\title{
Résumé
}

L'objectif de cette étude a été d'évaluer l'incorporation des résultats des recherches sur la gérance et la gestion des zones protégées du Risaralda. Pour cela, on a réuni l'information des recherches réalisées dans trois réserves nationales naturelles et trois réserves régionales naturelles. On a obtenu en tant que résultats que la plupart des études ont étés publiées par revues indexés et abordent les sujets des espèces végétales et animales. Tous les plans de gérance comptent avec un programme de recherche scientifique, et ils proposent neuf indicateurs rapportés à l'existence, l'actualisation, la recherche, la pertinence, l'incorporation du plan de gérance et le type de recherche. On conclut qu'il est fondamentale le renforcement des centres de documentation, la création de partenariats avec les universités et les instituts de recherche, la définition de lignes de recherche de chaque zone et appliquer un système d'indicateurs.

Mots-clés: indicateurs; recherche applique; conservation; priorités 


\section{Introducción}

El establecimiento de áreas protegidas se considera uno de los mecanismos primordiales para la conservación de los recursos naturales (Ramsay, 1990). Según la Unión Internacional para la Conservación de la Naturaleza (UICN) (2008), estas áreas se definen como "un espacio geográfico claramente definido, reconocido, dedicado y gestionado, mediante medios eficaces para conseguir la conservación a largo plazo de la naturaleza y de sus servicios ecosistémicos y sus valores culturales asociados" (Vásquez y Serrano, 2009). Generalmente, las áreas declaradas son importantes por contener comunidades bióticas funcionales, recursos genéticos, formas de vida y cuencas hidrográficas, que en su conjunto contribuyen al patrimonio natural de cada país (Vásquez y Serrano, 2009).

Con el fin de que las áreas protegidas cumplan con sus objetivos de conservación, es necesario realizar un manejo activo y efectivo a través de diferentes actividades. Una de ellas es la investigación, actividad fundamental para la toma de datos y por tanto para la generación de información para la toma de decisiones (Margolis y Salafsky, 1998). Generalmente, la investigación en las áreas protegidas tiene como prioridad el estudio del entorno físico-biótico, de su relación con la estructura social, económica y cultural y de las dinámicas involucradas en dicha relación (Sinisterra y Montenegro, 2005).

De esta forma, la investigación desempeña un papel vital en la gestión de las áreas protegidas y la conservación de su biodiversidad y servicios ecosistémicos. Por ende, es importante promover el establecimiento de líneas de comunicación entre los científicos y los administradores de las áreas, con el fin de incorporar el conocimiento a los mecanismos e instrumentos de gestión (Murcia y Kattan, 2009). Para lograr una efectiva relación entre conocimiento y gestión, las investigaciones deben responder a las necesidades de información de los gestores (Worboys et al., 2001), y según Murcia y Kattan (2009): "la información debe estar disponible y ser asequible y los manejadores del área deben tener la formación y el tiempo para extraer la información relevante e incorporarla en sus planes de acción”. Sin embargo, estos elementos no siempre son tenidos en cuenta; por ejemplo, Meijaard y Scheil (2007), al realizar una evaluación de la utilidad y los beneficios de las investigaciones para la conservación in situ de la fauna silvestre en Borneo, concluyen que en la mayor parte de los trópicos las investigaciones no se enfocaron en buscar las respuestas a los principales cuestionamientos de la biología de la conservación. Por esta razón, para hacer un manejo efectivo de las áreas se deben establecer unas prioridades claras de investigación y es por ello que los investigadores deben dar una mayor prioridad a abordar las necesidades y metas de conservación de las áreas protegidas (Primack, 2008).

Conforme con la tendencia mundial de establecimiento de áreas protegidas, Colombia cuenta con el Sistema Nacional de Áreas Protegidas (Sinap), conformado y en proceso de consolidación, gracias al compromiso adquirido por el país a través de la Política Nacional de Biodiversidad de 1995, la cual fue formulada con base en el Convenio de Diversidad Biológica suscrito por Colombia y ratificado a través de la Ley 165 de 1994. En el Decreto 2372 de 2010, por el cual se reglamenta esta ley, en relación con el Sinap y las categorías que lo conforman, se define a este sistema como "el conjunto de las áreas protegidas, los actores sociales e institucionales y las estrategias e instrumentos de gestión que las articulan, que contribuyen como un todo al cumplimiento de los objetivos generales de conservación del país".

Para la Unidad Administrativa Especial del Sistema de Parques Nacionales Naturales (UAESPNN), como componente y coordinador del Sinap, uno de los principales retos es la construcción de conocimiento, con el fin de sustentar la toma de decisiones para una gestión efectiva de las áreas protegidas. Para ello cuenta con instrumentos de gestión como los planes de manejo y de investigación. Este último contiene las líneas prioritarias, los programas y las acciones estratégicas en las cuales deben enmarcarse las investigaciones desarrolladas en las áreas protegidas. De este modo, se espera que la generación de conocimiento se base en las necesidades y los vacíos de información identificados en las líneas prioritarias que integran el plan de manejo del área (Sinisterra y Montenegro, 2005). 
Para la construcción de los planes de investigación, cada una de las áreas protegidas debe priorizar sus temas de investigación y posteriormente establecer sus líneas estratégicas; estas últimas deben estar enmarcadas dentro de las ocho líneas planteadas para la UAESPNN por Sinisterra y Montenegro (2005): 1) caracterización de ecosistemas, 2) dinámica de ecosistemas, 3) restauración ecológica, 4) uso de la biodiversidad y efecto del uso sobre poblaciones de vida silvestre, 5) percepción y valoración cultural del territorio, 6) institucionalidad, políticas y control social del territorio de las áreas protegidas, 7) valoración de biodiversidad y servicios ambientales y 8) impactos de políticas y obras de desarrollo. Para fortalecer el vínculo información-gestión, la UAESPNN ha identificado como primordial, además de desarrollar los planes de investigación, consolidar bases de datos con los resultados que se produzcan de dichos planes y divulgarlos.

Por otro lado, dentro de los componentes del Sinap se encuentran los Sistemas Regionales de Áreas Protegidas (Sirap), definidos en el Decreto 2372 del 2010 como subsistemas: "conjunto de áreas protegidas nacionales, regionales y locales, públicas o privadas existentes en las zonas que se determinan en la regionalización adoptada por este decreto, los actores sociales e institucionales y las estrategias e instrumentos de gestión que las articulan". Uno de los Sirap del país es el Sirap-Eje Cafetero, del cual hace parte el Sistema Departamental de Áreas Protegidas (Sidap) de Risaralda. El Sirap-ec cuenta con un plan de acción organizado en líneas estratégicas. En la línea de conocimiento, conservación y uso sostenible de los recursos naturales y la biodiversidad se han trabajado cuatro instrumentos, enfocados a incrementar los procesos de conocimiento e investigación: 1) la construcción de un sistema de información de la biodiversidad (sıв), 2) la generación de mecanismos de intercambio y capacitación, 3) la formulación de planes de manejo de especies focales y 4) la divulgación de resultados.

El presente estudio tuvo como objetivo compilar documentos sobre investigaciones realizadas en áreas protegidas de diferentes categorías entre los años 2000 y 2011 en Risaralda. Se escogió este departamento por el activo trabajo que se realiza en las áreas protegidas de esta zona en el marco del Sirap-EC, y, adicionalmente, se tuvo presente que la Corporación Autónoma Regional de Risaralda (Carder) cuenta con lineamientos generales para orientar las investigaciones que se realicen en sus áreas protegidas (Londońo, 2002). Complementando, la Carder, durante la vigencia 2007-2009, se adentró en el proceso de conformación de un grupo de investigación que le permitiera abordar, con carácter científico, los diferentes temas que la Corporación enfrenta en el ámbito de su quehacer institucional en el departamento de Risaralda (Plan de Acción 2007-2011).

\section{Materiales y métodos}

En primera instancia, se seleccionaron las áreas protegidas de diferentes categorías y administradas por autoridades del nivel nacional (UAESPNN) y departamental (CAR). Las áreas seleccionadas fueron tres parques nacionales naturales (PNN): PNN Los Nevados, PNN Tatamá y Santuario de Fauna y Flora (SFF) Otún-Quimbaya, y tres parques regionales naturales (PNR): PNR Ucumarí, PNR Alto del Nudo y PNR La Cuchilla del San Juan.

Posteriormente, se realizó una búsqueda de documentos sobre investigación y manejo en estas áreas entre los años 2000-2011, un intervalo de una década de análisis de la información existente. Para dicha recopilación se visitaron los centros de documentación de las áreas protegidas seleccionadas, la Carder, el Instituto Alexander von Humboldt y la UAESPnN. Se consultaron también bases de datos de las bibliotecas de la Universidad Nacional de Colombia, la Pontificia Universidad Javeriana y la Universidad de los Andes. Adicionalmente, se realizaron búsquedas en Google Académico y en base de datos como ISI, Science Direct y Scopus, entre otras. Como palabras clave se utilizaron: áreas protegidas, manejo, investigación, Risaralda y el nombre de cada uno de los parques.

Los documentos encontrados fueron organizados y clasificados en un base de datos con los siguientes campos: título, autor, año y entidad, tipo de publicación (libro, revista indexada, informe, tesis de grado 
o artículo no indexado), ubicación geográfica (parques seleccionados), contenido (investigación, planes de manejo, plan de investigaciones) y presencia o no de recomendaciones para el manejo y gestión del área. En el campo de investigación (contenido) se definieron dos categorías: especies y ecosistemas, y dentro de estas los estudios se clasificaron en subcategorías establecidas según Meijaard y Scheil (2007).

Para especies se definieron: 1) descriptivos (taxonomía, listas de especies, muestreos, morfología y bioquímica), 2) autoecológicos (movimiento de especies, hábitos alimenticios, hábitos reproductivos), 3) sinecológicos (interacciones entre grupos de especies o entre especies y su ecosistema), 4) amenazas (estudios que consideran explícitamente amenazas sobre las especies), 5) estudios de causa-efecto (investigaciones del cambio de poblaciones en relación con un factor especial), 6) estudios integrados, 7) estudios socioculturales y económicos y 8) planes de manejo de la especie. Para estudios sobre ecosistemas se definieron: 1) composición, 2) estructura, 3) función, 4) distribución espacial (conectividad, fragmentación, heterogeneidad), 5) restauración ecológica, 6) transformación, 7) valoración de bienes y servicios ambientales, 8) evaluación y monitoreo de impactos y capacidades de respuesta y 9) aspectos socioeconómicos y culturales. Para el análisis de los datos se empleó estadística descriptiva; posteriormente, las investigaciones encontradas también fueron clasificadas según las líneas estratégicas de investigación planteadas por Sinisterra y Montenegro (2005), mencionadas anteriormente.

Como complemento se analizó el contenido de los planes de manejo de las áreas estudiadas, en términos de vigencia, generación, recopilación y uso de información. Finalmente, se propuso una serie de indicadores que podrán ser usados en el futuro, al efecto de evaluar la incorporación de los resultados de las investigaciones al manejo y la gestión de las áreas protegidas de Risaralda. Estos indicadores fueron formulados teniendo en cuenta el análisis realizado en el presente estudio sobre las investigaciones y planes de manejo y experiencias a nivel regional y nacional.

\section{Resultados}

Se encontraron 266 documentos sobre investigaciones y manejo llevados a cabo en las áreas protegidas analizadas. La mayoría de los documentos corresponden al SFF Otún-Quimbaya $(n=89)$, seguido por el PNN Los Nevados $(\mathrm{n}=55)$, y las áreas con un menor número de documentos fueron el PNR Alto del Nudo $(n=3)$ y el PNR Cuchilla de San Juan $(n=1)$. En relación con el tipo de publicación, se encontró que la mayoría de documentos corresponden a artículos publicados en revistas indexadas (28\%). Sin embargo, esto no indica que este sea el tipo de publicación que predomine en cada una de las áreas protegidas analizadas (figura 1).

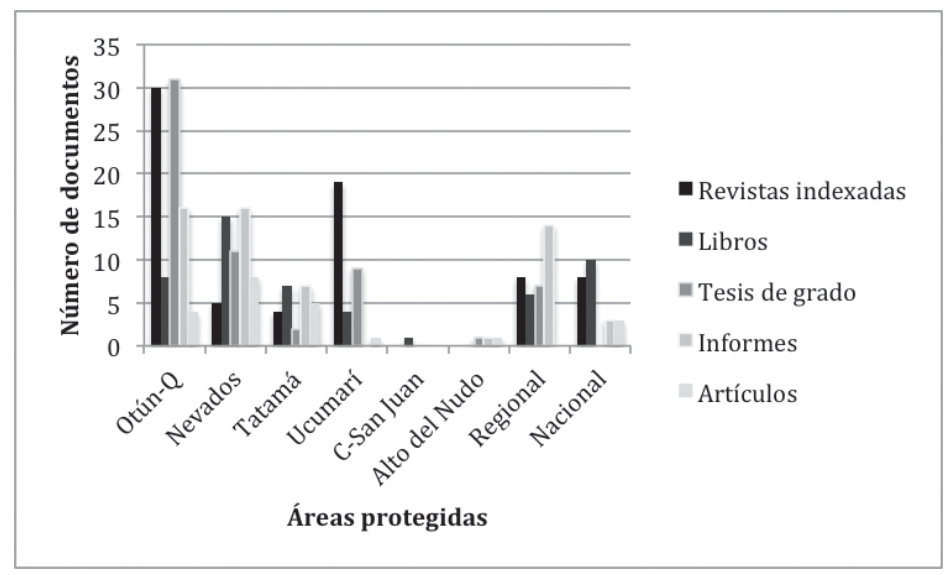

Figura 1. Clasificación de los documentos según el tipo de publicación 
En cuanto al contenido, el SFF Otún-Quimbaya contó con el mayor número de documentos clasificados como investigaciones $(\mathrm{n}=78)$, seguido por el PNN Los Nevados $(\mathrm{n}=36)$, el PNR Ucumarí y el PNN Tatamá. Con respecto a los documentos clasificados en la categoría de manejo, el pNN Los Nevados obtuvo el mayor número (30\%), seguido del SFF Otún-Quimbaya (22\%), el PNN Tatamá (10\%) y el PNR Ucumarí (6\%). Adicionalmente, existen estudios del nivel regional y nacional que abarcan las seis áreas protegidas analizadas. En el ámbito regional, veintidós documentos se clasificaron como investigaciones y once como documentos relacionados con el manejo, y en el orden nacional, veintiuno se clasificaron como investigaciones y tres como documentos relacionados con el manejo. Con respecto a los documentos de investigaciones, la mayoría fueron clasificados en la subcategoría de especies. Sin embargo, cabe anotar que en el PNN Los Nevados predominan los estudios sobre ecosistemas (figura 2).

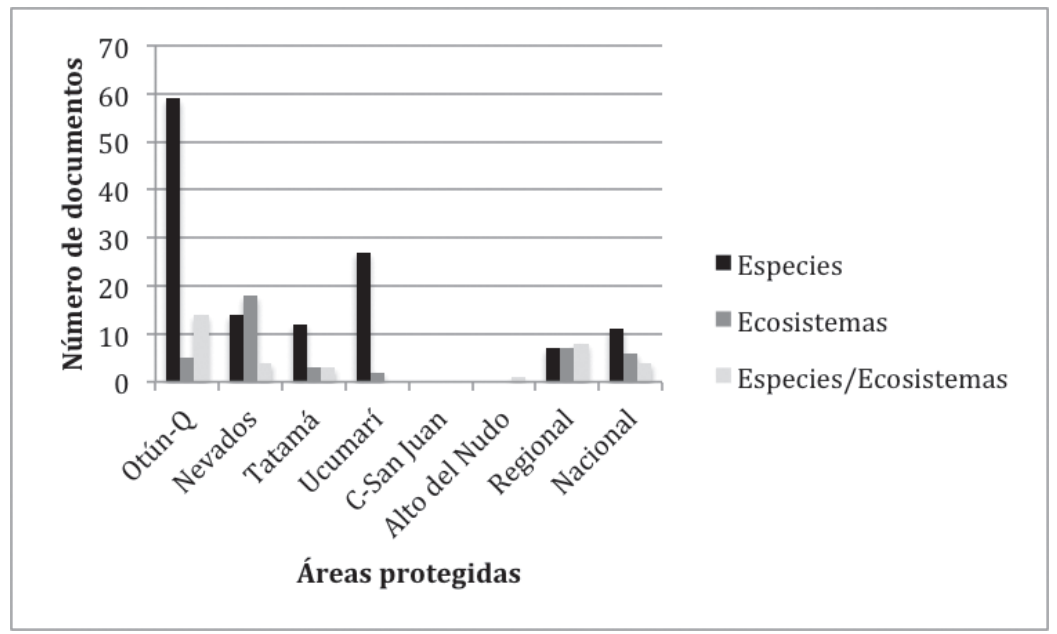

Figura 2. Clasificación de investigaciones según contenido (especies o ecosistemas)

Fuente: elaboración propia

Asimismo, se encontró que la mayoría de investigaciones sobre especies se relacionan con temáticas de índole descriptiva, sobre autoecología y sinecología. Las subcategorías con menor número de investigaciones fueron: estudios de causa-efecto, estudios integrados, estudios socioculturales y económicos, y planes de manejo de especies. Dentro de las investigaciones sobre ecosistemas, se evidencia que los tópicos más comunes son composición, estructura, distribución espacial y aspectos socioeconómicos y culturales. Las subcategorías con el menor número de investigaciones fueron: estudios sobre transformación, valoración de bienes y servicios ambientales y documentos sobre evaluación y monitoreo de impactos y capacidades de respuesta de los ecosistemas.

En relación con la presencia o no de recomendaciones para el manejo en los documentos, se obtuvo que 97 presentan recomendaciones (47,3\%), mientras que 108 no cuentan con ningún tipo de recomendación $(52,7 \%)$. Por último, en la clasificación de los documentos de acuerdo con los lineamientos estratégicos de investigación (figura 3), se encontró que la mayoría de trabajos (93) se ubican dentro de caracterización de ecosistemas, seguidos por los trabajos ubicados en la línea 2: dinámica de ecosistemas (32), y en la línea 3: restauración de ecosistemas (28). Las líneas con menos documentos fueron: valoración de biodiversidad y servicios ambientales e institucionalidad, y políticas y control social del territorio de las áreas protegidas. Algunos trabajos no fue posible ubicarlos dentro de las líneas de investigación, porque sus temas de investigación no entraban dentro de los tópicos que plantean los lineamientos; dentro de estos se encuentran veinte del SFF Otún-Quimbaya, veinte del PNR Ucumarí, siete del PNN Tatamá, cuatro del PNN Los Nevados, tres del ámbito regional y tres de orden nacional. Es importante tener en cuenta que los PNR no se rigen por los lineamientos de investigación de 
la UAESPNN, pero esta clasificación se realizó con el fin de comparar los contenidos de las investigaciones llevadas a cabo en las seis áreas.

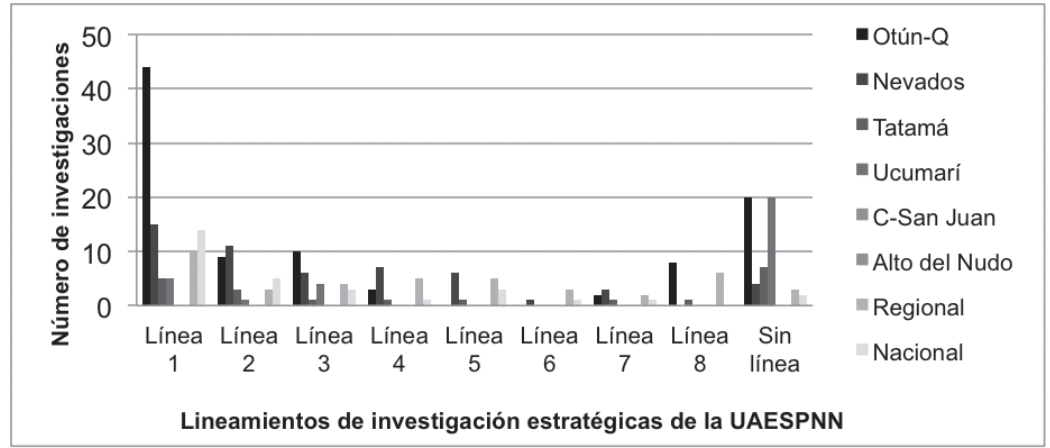

Figura 3. Estudios clasificados según los lineamientos de investigación para el Sistema de Parques Nacionales Naturales

\begin{abstract}
* Líneas de investigación: 1) caracterización de ecosistemas, 2) dinámica de ecosistemas, 3) restauración ecológica, 4) uso de la biodiversidad y efecto del uso sobre poblaciones de vida silvestre, 5) percepción y valoración cultural del territorio, 6) institucionalidad, políticas y control social del territorio de las áreas protegidas, 7) valoración de biodiversidad y servicios ambientales y 8) impactos de políticas y obras de desarrollo

Fuente: elaboración propia
\end{abstract}

Con referencia al análisis de los planes de manejo de cada una de las seis áreas protegidas (material suplementario), se encontró que todos mencionan los objetivos de conservación del área, objetivos específicos y metas (propósito del plan de manejo), y especifican la presencia de especies raras, amenazadas o endémicas; cuatro cuentan con inventarios de fauna y flora, evaluaciones del estado de los valores objeto de conservación $(\mathrm{VoC})$ y estudios de los atributos de sus poblaciones.

Se encontró que la mayoría de las áreas carecen de un inventario de bienes y servicios ambientales, a excepción del PNN Los Nevados y del PNR Cuchilla de San Juan. En relación con el componente operativo y normativo, los seis planes de manejo cuentan con un programa de investigación científica, han sido adoptados por la autoridad competente y se ha iniciado su implementación. Es importante mencionar que tres áreas: el PNN Los Nevados, el SFF Otún-Quimbaya y el PNR Ucumarí proponen que los resultados de investigación y monitoreo de los objetivos de conservación sean incorporados rutinariamente a la planificación.

\title{
Propuestas para reducir la brecha entre investigación y gestión
}

Para dar el contexto general de las propuestas dirigidas a reducir la brecha entre investigación y gestión, y como base de la propuesta de este estudio regional, se citan algunos estudios pertinentes realizados por otros autores. Para Pullin et al. (2004), los planes de manejo no contienen toda la información disponible generada en la investigación científica para sustentar la toma de decisiones, debido a la presión a la que se ven sometidos sus formuladores para ejecutar con urgencia las acciones, y también a que el tiempo no es suficiente para acceder a la información primaria necesaria para la construcción de los documentos.

Dicha conclusión puede relacionarse con los resultados de este estudio, en el cual se hace evidente la urgencia de crear mecanismos para incorporar los resultados de las investigaciones a los procesos de planificación, para la mayoría de áreas estudiadas, teniendo en cuenta además la calidad de las investigaciones. Pullin y Knight (2004), por ejemplo, proponen un modelo basado en evidencia de 
buena calidad sobre la cual basar las decisiones para la gestión, de modo que se permita realizar una revisión sistemática. En esta revisión "los trabajos de investigación seleccionados sobre la base de su relevancia son sometidos a una valoración crítica con un protocolo estándar" (Pullin et al., 2004), proceso en el que se tienen en cuenta variables como el diseño del estudio, la selección de temas, la recopilación de datos y el análisis.

En consecuencia, las investigaciones que no cumplen con el protocolo son rechazados o pasan a una segunda evaluación. En un trabajo anterior, Pullin y Knight (2001) presentaron una metodología para incorporar la investigación a la gestión, con base en dos principios fundamentales: 1) evaluar la literatura primaria y 2) hacer disponible la información para los tomadores de decisiones. Igualmente, consideran que los pasos básicos, a fin de crear una buena relación entre investigación y gestión son: 1) hacer preguntas que se puedan responder, 2) evaluar las investigaciones presentadas, 3) modificar en respuesta a la evidencia, 4) monitorear y evaluar la nueva acción y 5) difundir el conocimiento y el aprendizaje compartido. De este modo, se encontró que a nivel regional en el departamento de Risaralda se tienen retos relacionados con la incorporación de los resultados de las investigaciones al manejo y la gestión de las áreas protegidas. Esta necesidad incluye aspectos como la compresión y difusión de los resultados de las investigaciones, orientación de la investigación y actitudes de los tomadores de decisión (tabla 1).

Tabla 1. Práctica de la incorporación de la investigación a la gestión, con base en la evidencia

\begin{tabular}{|c|c|c|}
\hline Aspectos & Situación actual & Futuro \\
\hline $\begin{array}{l}\text { Comprensión de } \\
\text { los resultados de } \\
\text { la investigación }\end{array}$ & $\begin{array}{l}\text { Depende de la integración de la } \\
\text { información de los estudios de } \\
\text { investigación individuales }\end{array}$ & $\begin{array}{l}\text { Proporcionada por meta-análisis } \\
\text { y revisiones sistemáticas de la } \\
\text { investigación pertinente }\end{array}$ \\
\hline $\begin{array}{l}\text { Orientación de la } \\
\text { investigación }\end{array}$ & $\begin{array}{l}\text { Orientada por investigadores, } \\
\text { vinculados a agendas académicas }\end{array}$ & $\begin{array}{l}\text { Orientada por las necesidades } \\
\text { vinculadas a los lineamientos de } \\
\text { investigación }\end{array}$ \\
\hline $\begin{array}{l}\text { Resultados de la } \\
\text { investigación }\end{array}$ & $\begin{array}{l}\text { No hay seguimiento y evaluación } \\
\text { de la incorporación de los } \\
\text { resultados al manejo y gestión de } \\
\text { las áreas }\end{array}$ & $\begin{array}{l}\text { Propuesta de indicadores para } \\
\text { evaluar la incorporación }\end{array}$ \\
\hline $\begin{array}{l}\text { Los tomadores de } \\
\text { decisiones: } \\
\text { actitudes } \\
\text { con respecto a la } \\
\text { investigación }\end{array}$ & $\begin{array}{l}\text { Relativamente desinformados, con } \\
\text { falta de tiempo para la evaluación } \\
\text { de la investigación } \\
\text { y la interpretación }\end{array}$ & $\begin{array}{l}\text { Informados, acostumbrados a } \\
\text { utilizar y participar } \\
\text { en la investigación y su aplicación }\end{array}$ \\
\hline $\begin{array}{l}\text { Difusión de } \\
\text { resultados de la } \\
\text { investigación } \\
\text { (modo de acceso) }\end{array}$ & $\begin{array}{l}\text { Revistas, libros, informes, artículos } \\
\text { y tesis de grado }\end{array}$ & $\begin{array}{l}\text { Bases de datos en línea, resúmenes } \\
\text { de evidencia, directrices de manejo } \\
\text { y revisiones sistemáticas }\end{array}$ \\
\hline
\end{tabular}

Fuente: adaptado de Pullin y Knight (2003)

Por otra parte, según Sutherland et al. (2004), existe la necesidad de definir mecanismos para la revisión de la información biológica y hacer recomendaciones a los gestores. Para ello sugieren un formato web de base de datos que incluya la siguiente información: a) país, b) nombre del área, c) nombre y organización del contribuyente, d) categoría del hábitat, e) detalle del hábitat (por ejemplo, especies dominantes), f) tipo de problema, g) especies involucradas, h) acciones de conservación en sentido amplio, i) descripción detallada de la acción, j) consecuencias de la acción; con el propósito de presentar en forma sintética la información de la biodiversidad. En Colombia, particularmente, ya se cuenta con un sistema de información sobre biodiversidad (sів) que facilita la gestión de datos e información y apoya oportuna y eficientemente procesos de investigación, educación o toma de decisiones relacionadas con el conocimiento, la conservación y el uso sostenible de la diversidad biológica del país. 
Según Roux et al. (2006), la mejor manera de reducir la brecha entre investigación y gestión es crear las condiciones adecuadas para el flujo de conocimiento entre investigadores, responsables políticos y administradores de recursos. De manera específica, se propone que exista una coproducción de conocimiento donde el flujo de información sea un proceso bidireccional entre la ciencia y la administración, para garantizar la aplicación y adopción del nuevo conocimiento (Roux et al., 2006). De acuerdo con lo anterior, para Hall y Fleishman (2010): "cuando los usuarios finales puedan evaluar explícitamente si un nuevo método permite alcanzar objetivos de manejo, ahorrar dinero y reducir el riesgo bajo incertidumbre, la comunidad profesional habrá tendido un puente entre la investigación y la aplicación" (p. 126). Knight y Cowling (2006), por su parte, proponen un mecanismo para superar la brecha entre investigación y aplicación que existe entre la ciencia y el manejo del bioma Thicket (bioma que conforma las tierras áridas). Esta iniciativa reúne a investigadores, ejecutores (como los propietarios de tierras) y facilitadores (funcionarios del gobierno o administrativos) cada año para discutir e identificar problemas de investigación y orientaciones de gestión. De este modo, en el caso colombiano podría aplicarse un foro semejante para cada uno de los seis biomas propuestos por Forero y Joppa (2010), o bien para las regiones definidas en el Decreto 2372, el cual se convertirá en un mecanismo de comunicación entre los actores involucrados en el manejo de las áreas protegidas.

Murcia y Kattan (2009), por su parte, resaltan la urgencia de establecer una línea de aplicación de la ciencia al manejo de las áreas protegidas. En este sentido, proponen mecanismos para que los científicos sean conscientes de las necesidades de información de estos espacios y de los administradores, y para que los gestores sepan cómo acceder, procesar e incorporar la información pertinente. Los mecanismos propuestos son: diálogos activos entre las partes, traductores de ciencia en la Academia, el Gobierno y las organizaciones no gubernamentales, y ejecución de proyectos conjuntos. En Colombia, el Decreto 2372 de 2010, artículo 8 establece subsistemas de gestión de áreas protegidas: subsistemas regionales y subsistemas temáticos, los cuales permitirán una articulación entre actores sociales e institucionales para establecer estrategias e instrumentos de gestión en las áreas protegidas.

De acuerdo con los resultados encontrados y las propuestas de otros estudios, es importante tener una propuesta de indicadores para la región, que permita analizar la relación entre investigación y gestión en las áreas protegidas. Como primer paso se proponen una serie de indicadores cualitativos (tabla 2) y cuantitativos (tabla 3) que permitirán evaluar cómo se encuentra el área en relación con sus instrumentos guía de manejo y de investigación. Igualmente, permitirán inferir el grado de incorporación de los resultados de las investigaciones al manejo y gestión de las áreas protegidas. Se propone que estos indicadores sean medidos cada cinco años, periodo en el que se formulan o actualizan los planes de manejo. Su aplicación hará posible identificar vacíos de conocimiento, comparar entre prioridades de manejo e información existente y analizar en qué estado se encuentra la información disponible.

Tabla 2. Indicadores cualitativos

\begin{tabular}{lll}
\hline Factor & Indicador & Rango \\
\hline Existencia & ¿El área protegida cuenta con un plan de manejo? & Sí/No \\
\hline Actualización & $\begin{array}{l}\text { ¿El plan de manejo ha sido actualizado al menos cada cinco } \\
\text { años? }\end{array}$ & Sí/No \\
\hline Investigación & $\begin{array}{l}\text { ¿El plan de manejo cuenta con un plan o programa de } \\
\text { investigaciones? }\end{array}$ & Sí/No \\
\hline Pertinencia & $\begin{array}{l}\text { ¿El plan de manejo especifica los lineamientos de investigación } \\
\text { del área protegida? }\end{array}$ & Sí/No \\
\hline
\end{tabular}


Tabla 3. Indicadores cuantitativos

\begin{tabular}{|c|c|c|}
\hline Factor & Indicador & Rango \\
\hline \multirow{4}{*}{ Incorporación } & & 0,81-1 Excelente \\
\hline & \# doc citados en el Plan de Manejo & 0,51-0,8 Bueno \\
\hline & \# doc encontrados para el área & 0,31-0,5 Regular \\
\hline & & 0-0,3 Deficiente \\
\hline Tipo de información & $\frac{\text { \# doc según tipo de publicación }}{\text { \# doc encontrados para el área }}$ & $0-1$ \\
\hline \multirow{2}{*}{$\begin{array}{l}\text { Contenido de la } \\
\text { investigación citada }\end{array}$} & $\begin{array}{l}\text { \# doc citados en el PM sobre especies } \\
\text { \# doc citados en el Plan de Manejo }\end{array}$ & $0-1$ \\
\hline & $\begin{array}{l}\text { \# doc citados en el PM sobre ecosistemas } \\
\text { \# doc citados en el Plan de Manejo }\end{array}$ & $0-1$ \\
\hline Lineamientos & $\begin{array}{l}\text { \# doc citados en el PM según lineamiento } \\
\text { \# doc citados en el Plan de Manejo }\end{array}$ & $0-1$ \\
\hline Fuente de información & $\begin{array}{l}\text { \# recomendaciones o propuestas contenidas } \\
\text { en el plan de manejo que tienen citación de } \\
\text { literatura }\end{array}$ & $0-\alpha$ \\
\hline
\end{tabular}

*Abreviaturas: doc: documentos, PM: Plan de Manejo, sP: especies. Lineamientos: líneas estratégicas de investigación formuladas para el Sistema de Parques Nacionales Naturales, planteadas por Sinisterra y Montenegro (2005)

Fuente: elaboración propia

\section{Discusión}

\section{Análisis de las investigaciones recopiladas}

Mediante el análisis de los resultados obtenidos de la recopilación y análisis de los documentos sobre investigación y manejo de las áreas protegidas de Risaralda, se logró establecer cuáles son las temáticas comunes de las investigaciones de las áreas protegidas estudiadas, el nivel de acceso a estos estudios y su aplicación al manejo de las áreas.

En términos de ubicación geográfica, se encontró que el SFF Otún-Quimbaya cuenta con el mayor número de documentos. Esto se debe, posiblemente, a las particularidades del área, dentro de las que se encuentran su reducido tamaño, la presencia de la Fundación EcoAndina hasta el 2007 y un permiso marco otorgado al Centro de Investigaciones y Estudios en Biodiversidad y Recursos Genéticos (Ciebreg) desde el año 2006 para realizar investigaciones en dicho santuario (Castiblanco, 2009). Por el contrario, el menor número de documentos se encontró en los PNR Alto del Nudo y Cuchilla de San Juan, debido posiblemente a los pocos incentivos que existen para promover los procesos de investigación que generen conocimiento y permitan realizar una valoración de la biodiversidad y los demás servicios ambientales. Además, no se han definido los $\mathrm{VoC}$ de conservación para los dos PNR Cuchilla de San Juan y Alto del Nudo (Londoño, 2011a; Londoño, 2011b). Hoy en día el pNr Cuchilla de San Juan solamente cuenta con información sobre aves y plantas vasculares de parques municipales que se encuentran en sus límites, y en el caso del PNR Alto del Nudo únicamente existe un inventario de avifauna (Londońo, 2011a; 2011b).

Con base en los resultados obtenidos se puede evidenciar que ha sido mayor la investigación realizada en los parques nacionales (193) que en los regionales (73), fenómeno que puede ser explicado, 
en parte, porque el Sirap-EC no cuenta todavía con un plan de investigaciones que complemente los lineamientos de investigación de las áreas de la UAESPNN y que se aplique en las áreas de categoría regional y municipal.

La categoría de tipo de publicación permitió evaluar el acceso a la información de cada una de las áreas. Se encontraron dos situaciones que dificultan la consulta de todos los documentos existentes: la primera, la dispersión de la información, y la segunda está relacionada con el tipo de publicación. Dado que gran parte de las investigaciones se encuentran en revistas indexadas, su contenido no es accesible para el público en general. En el caso específico del centro de documentación del SFF Otún-Quimbaya se encontró que además de una base de datos en formato electrónico, existen listados elaborados en años anteriores al 2010 que evidencian que muchos de los estudios realizados no se encuentran en los sitios consultados y no reposan en los respectivos centros de documentación.

Con respecto al contenido de los documentos, se encontró que la mayoría de los trabajos científicos se enfocan en el estudio de especies $(63,4 \%)$ y son pocos los estudios sobre ecosistemas, valoración de bienes y servicios ambientales, evaluación y monitoreo de impactos y los relacionados con aspectos socioeconómicos y culturales, los cuales son de vital importancia para la gestión en las áreas con presencia humana, así como de sus zonas de influencia (Castiblanco, 2009). Este hecho puede deberse a que las investigaciones tienden a realizarse sobre los $\mathrm{VoC}$ de conservación del nivel de especie y que los programas de investigación de las áreas estén enfocados en generar mayor conocimiento de estos.

\section{Líneas estratégicas de investigación}

Con referencia a los lineamientos de investigación para la UAESPNn (Sinisterra y Montenegro, 2005), el elevado número de documentos clasificados en la línea de caracterización de ecosistemas, refleja en gran parte que las investigaciones se han enfocado en su mayoría en el estudio de los valores/ objeto de conservación: pava caucana (Penelope perspicax), oso de anteojos (Tremarctos ornatus), guagua loba (Dinomys branickii), etc., ya sea a nivel de especie o de ecosistema.

Por lo tanto, debe ser una prioridad que los investigadores especifiquen en cuál lineamiento de investigación se enmarca su estudio, todo con el propósito de presentar al público de forma integrada y coherente los aspectos biofísicos y sociales de las áreas (Arango, 2005), así como establecer unos ejes de ordenamiento del conocimiento (Sinisterra y Montenegro, 2005), entendidos como definición y construcción de las líneas prioritarias de investigación, construcción de sus objetivos y diseño de programas para desarrollarlas. Estos ejes conllevan una priorización de los temas de investigación y se dará uso pertinente de los resultados de las investigaciones en un aspecto específico del manejo y gestión de cada una de las áreas.

De acuerdo con el análisis realizado a los planes de manejo de cada una de las seis áreas, en el SFF Otún-Quimbaya, en el PNN Nevados y en el PNN Tatamá, dentro de sus objetivos y prioridades en el plan de manejo se encuentra como meta a cinco ańos la elaboración de un documento que contenga las líneas de investigación definidas para el área. En el PNR Ucumarí, en particular, se pretende desarrollar una línea de investigación sobre la valoración de los servicios ambientales prestados por el parque; este fue uno de los objetivos que tuvo mayor cumplimiento en este parque regional (Londońo et al., 2011).

\section{Relación investigación-manejo}

El plan de manejo se considera un elemento clave para el cumplimento de los objetivos de conservación de las áreas. En este documento se fijan los objetivos de trabajo y se proponen medidas para alcanzarlos en un intervalo de tiempo específico (Pullin y Knight, 2003). Además, se convierte en un instrumento de apoyo para la toma de decisiones, dado que pretende identificar la insuficiencia de la información y las áreas donde se requiere una mayor investigación científica (Pullin y Knight, 2003). 
Los planes de manejo son fundamentales para establecer las prioridades de investigación, el plan de acción y seguimiento de sus objetivos de conservación (Pullin y Knight, 2001). De este modo, para la formulación de los planes de manejo el proceso de recopilación y revisión de investigaciones sobre la biodiversidad de las áreas se considera un componente fundamental (Sutherland et al., 2004). En el presente estudio se encontró que en dos de las seis áreas los planes de manejo están desactualizados (PNR Ucumarí [1999-2000] y PNR Cuchilla de San Juan [2001]). Es importante resaltar que el PNR Ucumarí ha sido considerado, dentro de las áreas protegidas del Sidap Risaralda, como pionero en la investigación de la biodiversidad (Londoño et ál., 2011); por lo tanto, es necesario actualizar su plan de manejo.

En relación con incorporar los resultados de las investigaciones a la planificación de las áreas, el PNN Nevados y el sfF Otún-Quimbaya establecen dentro de su plan de manejo la necesidad de consolidar un plan de investigaciones que cuente con el apoyo de las universidades regionales y los institutos de investigación. Con respecto al PNR Ucumarí, en su plan de manejo se encontró que el programa de investigación ha sido el más exitoso de la región, dado su rigor científico y el que su objetivo principal sea impulsar el diseño y la ejecución de un programa de investigación en biología de la conservación que genere información necesaria para el manejo efectivo del área. De este modo, todas las áreas analizadas identifican en su plan de manejo como prioridad establecer un plan que enmarque y guie las próximas investigaciones dentro de unas líneas definidas.

\section{Conclusiones}

En Colombia existen retos relacionados con la incorporación de los resultados de las investigaciones al manejo y gestión de las áreas protegidas. Asimismo, la articulación entre Academia y manejo del área protegida depende directamente de los resultados de las investigaciones realizadas. En el caso del SFF Otún-Quimbaya y del PNN Los Nevados se encuentra en consolidación un plan de investigaciones, así como en el caso del Sirap Eje Cafetero. Sin embargo, se evidencian las siguientes dificultades: 1) descentralización de la información, lo cual dificulta la consulta de todos los documentos existentes, 2) la mayoría de las investigaciones se han realizado sobre especies, y 3) carencia de trabajos en los tópicos de ecosistemas y aspectos sociales, económicos y culturales a nivel de especie. Lo anterior evidencia que los estudios no están respondiendo a todas las líneas de investigación planteadas por la UAESPNN. Se encontró que la mayoría de los trabajos están asociados a la primera línea: caracterización de ecosistemas, realidad que refleja que gran parte de las investigaciones tratan sobre el estudio de los valores/objeto de conservación, y no cuentan con un inventario de bienes y servicios ambientales en su plan de manejo.

Resulta primordial la incorporación de los resultados de las investigaciones al manejo de las áreas protegidas, la generación de vínculos estrechos entre la investigación y la práctica, la realización de investigaciones enmarcadas dentro de los lineamientos de cada área y el establecimiento de métodos para hacer la información más accesible.

Por lo anterior, se propone lo siguiente: 1) fortalecer los centros de documentación de cada una de las áreas protegidas, que cuenten con una copia de todas las investigaciones realizadas y con una base de datos actualizada que las recopile; 2) establecer alianzas con universidades e institutos de investigación, con el fin de ampliar y profundizar el conocimiento de la biodiversidad en cada uno de las áreas, además con el propósito de crear estaciones biológicas; 3) definir las líneas de investigación de cada área y los vacíos de conocimiento; y 4) aplicar un sistema de indicadores que evalúe periódicamente la incorporación de los resultados de las investigaciones al manejo y gestión de las áreas. Adicionalmente, es de vital importancia hacer un llamado de atención a las autoridades ambientales en Colombia para que incluyan este tipo de estudios académicos dentro de su política de biodiversidad actual, con el fin de tener experiencias sobre las áreas protegidas. 


\section{Referencias}

Arango, N. (Ed.). (2005). Bases para el diseño de sistemas regionales de áreas protegidas. Bogotá: Instituto de Investigación de Recursos Biológicos Alexander von Humboldt.

Castiblanco, A. J. (2009). Análisis de la base de datos de investigación y recomendaciones para el desarrollo de procesos de investigación en las áreas protegidas de la DTNO (manuscrito no publicado).

Forero, M. G. y Joppa, L. (2010). Representation of Global and National Conservation Priorities by Colombia’s Protected Area Network. Plos One, (5), 1-11.

Hall, J. A. y Fleishman, E. (2010). Demonstration as a Means to Translate Conservation Science into Practice. Conservation Biology, 24, 120-127.

Knight, A. T. y Cowling, R. M. (2006). Into the Thick of it: Bridging the Research-Implementation Gap in the Thicket Biome through the Thicket Forum. South African Journal of Science, (102), 406-408.

Londoño, E. (2002). Áreas naturales protegidas de Risaralda. Pereira: Corporación Autónoma Regional de Risaralda. Londońo, E., Nadachowski, E. y Valencia, M. (2011). Evaluación de la efectividad del manejo para el Sistema Departamental de Áreas Protegidas de Risaralda Cuadernos de trabajo No 2. Pereira: Sistema Departamental de Áreas Protegidas de Risaralda.

Londońo, E. (2011a). Evaluación de la efectividad del manejo para el Sistema Departamental de Áreas Protegidas de Risaralda. Cuadernos de trabajo N 5. Pereira: Sistema Departamental de Áreas Protegidas de Risaralda.

Londoño, E. (2011b). Evaluación de la efectividad del manejo para el Sistema Departamental de Áreas Protegidas de Risaralda. Cuadernos de trabajo Nº . Pereira: Sistema Departamental de Áreas Protegidas de Risaralda.

Margolis, R. y Salafsky, N. (1998). Measures of Success Designing, Managing, and Monitoring Conservation and Development Projects. Washington, D.C: Island Press.

Meijaard, E. y Scheil, D. (2007). Is Wildlife Research Useful for Wildlife Conservation in the Tropics? A Review for Borneo with Global Implications. Biodiversity and Conservation, (16), 3053-3065.

Murcia, C. y Kattan, G. (2009). Application of Science to Protected Area Management: Overcoming the Barriers. Annals of the Missouri Botanical Garden, (96), 508-520.

Primack, R. B. (2008). Fundamentos de conservación biológica perspectivas latinoamericanas. México, D. F.: Fondo de Cultura Económica.

Pullin, A. y Knight, T. (2001). Effectiveness in Conservation Practice: Pointers from Medicine and Public Health. Conservation Biology, (15), 50-54.

Pullin, A. y Knight, T. (2003). Support for Decision Making in Conservation Practice: An Evidence-Based Approach. Journal for Nature Conservation, 11, 83-90.

Pullin, A., Knight, T., Stone, D. y Charman, K. (2004). Do Conservation Managers Use Scientific Evidence to Support their Decision-Making? Biological Conservation, (119), 245-252.

Ramsay, J. (1990). Manejo de áreas protegidas en los trópicos. Gland: International Union for Conservation of Nature (IUCN).

Roux, D. J., Rogers, K. H., Biggs, H. C., Ashton, P. J. y Sergeant, A. (2006). Bridging the Science-Management Divide: Moving from Unidirectional Knowledge Transfer to Knowledge Interfacing and Sharing. Ecology and Society, (11), 1-20.

Sinisterra, J. y Montenegro, M. (2005). Formulación de planes de investigación en Parques Nacionales Naturales. Bogotá: Panamericana Formas e Impresos. 
Sutherland, W. J., Pullin, A. S., Dolman, P. M. y Knight, T. M. (2004). The Need for Evidence-Based Conservation. Trends in Ecology and Evolution, (19), 305-308.

Vásquez, V. H. y Serrano, G. (2009). Las áreas naturales protegidas de Colombia. Bogotá: Conservación Internacional Colombia, Fundación Biocolombia.

Worboys, G., Lockwood, M. y De Lacy, T. (2001). Protected Area Management Principles and Practice. Nueva York: Oxford University Press. 